\title{
Acknowledgement of manuscript reviewers 2018
}

\section{Victoria G. Vivilaki ${ }^{1}$}

\section{CONTRIBUTING REVIEWERS}

The editors of European Journal of Midwifery would like to thank all our reviewers who have contributed to the journal in Volume 2 (2018).

\section{AFFILIATION}

1 Department of Midwifery, University of West Attica, Aigaleo, Athens, Greece

\section{CORRESPONDENGE TO}

Victoria G. Vivilaki. Department of Midwifery,

University of West Attica, Passions 44-46,

11631 Athens, Greece.

E-mail:v.vivilaki@gmail.com

\section{Cathy Ashwin \\ United Kingdom \\ Carrie Bonsack}

United States

\section{Susannah Brady}

Australia

Jim Brigagão

Brazil

\section{Irene Calvert}

New Zealand

\section{Megan Cooper}

Australia

\section{Abdolreza Daraei}

Iran

\section{Sarah Davies}

United Kingdom

Rafael del Rosal

Spain

\section{Ramón Escuriet}

Spain

Gracia Fellmeth

United Kingdom

\section{Sarah Fogarty}

Australia

\section{Hajdu Gabor}

Hungary

\section{Mahin Gheibizadeh \\ Iran}

\section{Enrique Gomez-Pomar \\ United States}

\section{Wendy Hall}

Canada

\section{Tutik Hariyati}

Indonesia

\section{Caroline Homer}

Australia

Hyuk Im

South Korea

Lara Jansiski Motta

Brazil

Ingrid Jepsen

Denmark

Garg Kamakshi

India

\section{Annika Karlstrom \\ Sweden}

\section{Bengt Kayser \\ Switzerland}

Martina Koenig-Bachmann
Austria

Sklempe Kokic

Croatia

\section{Mario Lopes \\ Portugal}

\section{Aidalina Mahmud \\ Malaysia}

\section{Daniel Martingano}

United States

\section{Robyn Maude}

New Zealand

Lois McKellar

Australia

\section{Polona Mivšek}

Slovenia

Stephanie Morgan

United States 
Tiina Murto

Finland

\section{Rhona O'Connell}

Ireland

\section{Lucinda Roper}

Australia

\section{Shiri Sadeh-Sharvit}

United States

Paula Santos

Portugal

Kirti Saxena

India
Katja Schrøder

Denmark

Elisabeth Severinsson

Norway

\section{Susanne Simon}

Germany

Jen Stevens

United States

Seyed Saeed Tabatabaee Iran

Melania Tudose

Romania
Deborah Turnbull

Australia

Joeri Vermeulen

Belgium

Victoria Vivilaki

Greece

Therese Wiegers

Netherlands

Xianglong $\mathrm{Xu}$

China 